\title{
Navigation Application using Recycler View
}

\author{
Mukul Gupta ${ }^{1}$ Dr. Vinay Kumar Saini ${ }^{2}$ \\ 1B. Tech Scholar, Department of IT, Maharaja Agrasen Institute of Technology, Delhi, India \\ ${ }^{2}$ Assistant Professor, Department of IT, Maharaja Agrasen Institute of Technology, Delhi, India
}

To Cite this Article

Mukul Gupta and Dr. Vinay Kumar Saini, "Navigation Application using Recycler View", International Journal for Modern Trends in Science and Technology, Vol. 07, Issue 01, January 2021, pp.-31-35.

Article Info

Received on 22-November-2020, Revised on 18-December-2020, Accepted on 22-December-2020, Published on 28-December-2020.

\section{ABSTRACT}

Whenever we visit an amusement park or theme park or water park or a zoo, a lot of our time is wasted on navigating throughout the park and we get tired of roaming around. It would be a much better experience for the user if there was a personalized mobile application that would help the users navigate through the park easily. This particular application is meant for National Zoological park or The Delhi Zoo located in Delhi, India. This application can significantly enhance the experience of the people visiting the national zoological park by helping them keep track of the animals they have visited and guiding them to a particular animal with just a single click.

This application is meant for android devices and it has two lists which were implemented using RecyclerView. Android recyclerview is the most advanced version of the listview. Basically, an android listview is used to present a simple data set. If you want to display large data set in your app, you should use recyclerview. RecyclerView is a much more powerful, flexible, smooth, and a major enhancement of listview.

KEYWORDS: navigate, android application, recyclerview, listview

\section{INTRODUCTION}

Every one of us likes to visit amusement parks, water parks, theme parks, zoo, etc... but it's only after we reach there that we realize that it is very tiring to roam around the park. Also, we get frustrated trying to navigate through the park. This all takes up most of our effort and we are not able to indulge in the entire experience because we are frustrated or tired. To make the user experience better, there should be a personalized mobile application for these attractions. This application is inspired by my own personal experience of visiting the National Zoological Park or The Delhi Zoo. When I visited the zoo, there was no way to keep track of the animals you have already visited or which path to take to the place you would like to visit next. There was just a guide map (shown below) which was of no practical use because I couldn't tell which direction I was facing and which path was which.

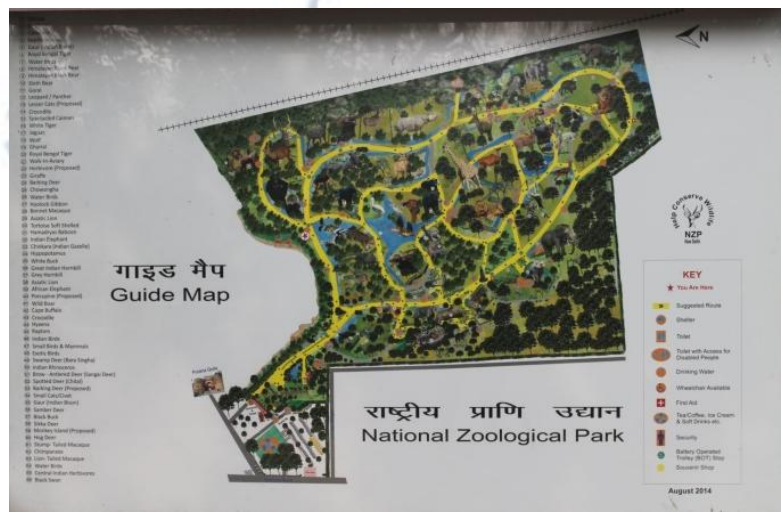

Figure 1: Guide Map 
Also, in Google Maps, the national zoological park is just one big patch of grass (shown below) like any other park. So, I created this application which will make the experience of zoo visitors much better and save a lot of effort on their part.

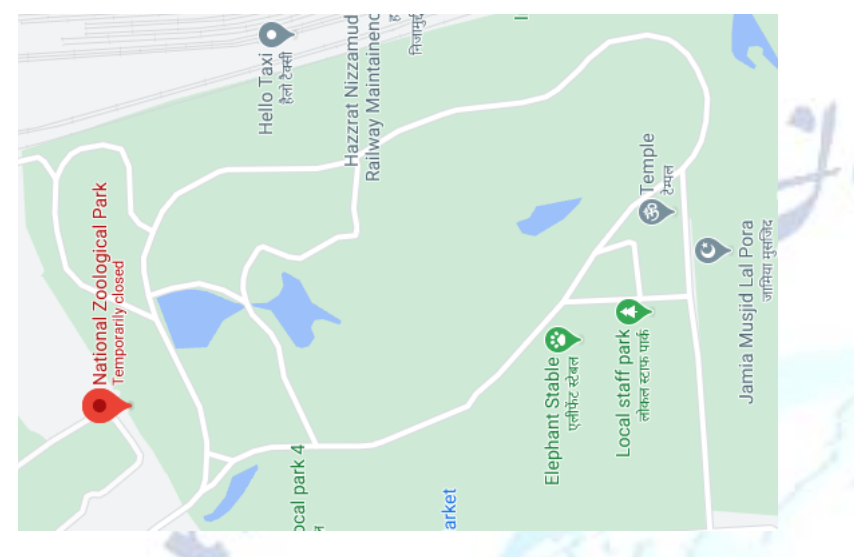

Figure 2: National Zoological Park on Google Maps

This application in meant for people visiting the National Zoological Park or the Delhi Zoo. Every person visiting the National Zoological Park can benefit from this application.

This application will significantly reduce the effort on the part of the user while at the same time making their experience far more enjoyable.

The Android Application that I created is curated for National Zoological Park only but the same methodology can be followed for almost any Amusement Park, Theme Park, Water Park, Zoo, etc... Having a personalized application for your own Amusement Park, Theme Park, Water Park, Zoo, etc. is a great way to make the experience of the visitors better.

\section{INTRODUCTION TO RECYCLERVIEW}

Android recyclerview is the most advanced version of the listview. Basically, an android listview is used to present a simple data set. If you want to display large data set in your app, you should use recyclerview. RecyclerView is a much more powerful, flexible, smooth, and a major enhancement of listview.

Previously most of the developers used android Listview and android Gridview. according to the android developer web site, recyclerview is initiated with Android Lolipop, the version is 22.1.0 which is under the support library. And it belongs to Maven artifact

(com.android.support:recyclerview-v7:28.0.0-alph a1). The latest version of the RecyclerView(androidx.recyclerview:recyclerview: 1 .2.0-alpha01) is related to AndroidX .

RecyclerView has more attractive, smooth controls, functionality, and reliability over list view items. Recyclerview is using a significant design pattern to render the result. There are more advantages than using other views such as ListView, GridViews. Recyclerview is much more customizable than listview and gives a lot of control and power to its developers.

\section{ADVANTAGES OF USING RECYCLERVIEW}

\section{Performance on Loading}

The major advantage of the usage of RecyclerView is the performance when loading list items in the list to the view. RecyclerView prepares the view behind and ahead beyond the visible entries. It gives significant performance when you need to fetch the bitmap image in your list from a background task. If you use RecyclerView.setHasFixedSize, it will give you a dramatically faster way. ListView is based on the premise that there is no way to precalculate or cache the size of entries in the list until completion of the scrolling.

\section{ViewHolder Pattern}

This is the major difference between the listview and recyclerview. It is recommended to use the ViewHolder design pattern but is not compulsory. However, it is mandatory using RecyclerView.ViewHolder class. When we use listview we faced a lot of problems that are solved in recyclerview and it is a bit more complex than other views' implementations.

\section{Layout Manager}

This is another major enhancement of the recyclerview than listview. In Listview, there is only one type of view available. There is no optimum way to implement for the horizontal list view. Using recyclerview we can have a different layout with layout managers and can be done all the views dynamically. Those are as follows.

- LinearLayoutManager - supports for vertical and horizontal lists

- GridLayoutManager - supports for Grid view lists. (ex:- Image Gallery) 
- StaggeredLayoutManager - supports for staggered lists (ex:-Pinterest)

\section{Item Decoration}

In ListViews it is harder to implement by adding borders or dividers and adding different decorations to list items. But in the case of RecyclerView is RecyclerView.ItemDecorator class gives more sophisticated control to the developer. However, it is a bit more time consuming and complex.

\section{Item Animator}

Listview does not give much support for having good animations. But, RecyclerView brings a whole new dimension to it. Using the RecyclerView.ItemAnimator class, animating the views becomes so much easy and intuitive.

\section{OnItemTouchListener}

RecyclerView gives much more powerful control to developers via RecyclerView.OnItemTouchListener than ListView. But it has more implementation to complete by the developer. Although list item click events in ListView can be handled through the AdapterView.OnItemClickListener Interface.

\section{DISADVANTAGES OF USING RECYCLERVIEW}

1. More complex than ListView.

2. Take much more time to implement than ListView implementation.

3.It can take a lot of time for a beginner to understand a RecyclerView fully.

4.It can unnecessarily make your coding life difficult.

\section{IMPLEMENTING ANDROID APPLICATION}

\section{Labeling locations of all animals on Google maps}

This step included me marking locations of all animals on Google Maps. For this, I visited the National Zoological Park and spent the entire day there marking locations of animals. I went to see every animal one by one and labeled that particular location on Google maps accordingly. Below is the image on my Google Maps Application after marking all the locations of the animals.

I marked a total of 57 animals. Some animals were present at more than one location, so the one which came second in the suggested path, was marked with a "(2)" next to it. For eg, the Asiatic lion which came first was marked as "Asiatic Lion" and the one which came second was marked as "Asiatic Lion (2)".

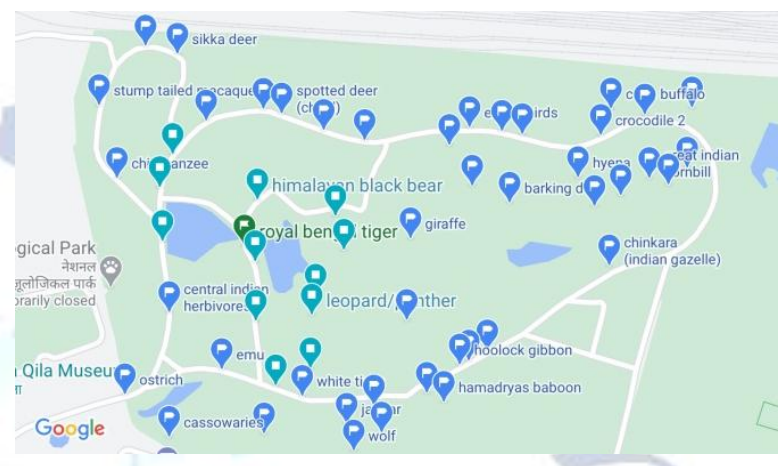

Figure 3: Google Maps snapshot with all locations labeled

\section{UI of the Application}

The UI of the application consists of two lists namely - A-Z and Route Order. In each list, all the animals are listed, one in alphabetical order and the other in the order in which they appear. The two lists are in the form of tabs. Also, in each list there is a check-box next to the name of each animal to mark them as visited. After checking a particular box, a message pops up that tells the user that that particular animal has been marked as visited. For example, if user clicks on the check-box next to Cassowaries, a message will pop-up and say "Cassowaries marked as visited".

When a user clicks on any animal's name, Google Maps is launched directing the user to the location of that animal.

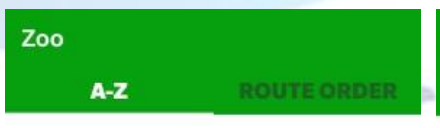

AFRICAN ELEPHANT

ASIATIC LION

ASIATIC LION (2)

BARKING DEER

BLACK BUCK

BONNET MACAQUE

BROW-ANTLERED DEER (SANGAI DEER)

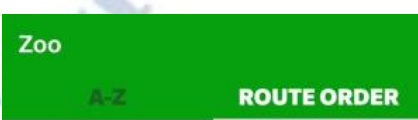

OSTRICH

EMU

CASSOWARIES

REPTILE HOUSE

GAUR (INDIAN BISON)

ROYAL BENGAL TIGER

WATER BIRDS

HIMALAYAN BLACK BEAR 


\section{Adding functionality to the application using RecyclerView}

To add functionality to the application, the following Java files were created in the com.example.zoo folder of the project which exists in the java folder. To achieve functionality, Fragments were added in RecyclerView.

- ViewPagerAdapter.java : This java class was used to create two different tabs.

- MainActivity.java : This java files creates the home of the application and add two tabs to the homepage which are 'A-Z' and 'Route Order' using class ViewPagerAdapter.java.

- Contact.java : This is a simple java class which defines the structure of each item. It has a constructor, a getter and a setter.

- Fav.java : This is a simple java class which defines the structure of each item. It has a constructor, a getter and a setter.

- FragmentContact.java : The layout of the A-Z order list was achieved using this class.

- FragmentFav.java : The layout of the Route Order list was achieved using this class.

- RecyclerViewAdapter.java : This java class forms the recycler view for the A- $Z$ list. This is the class that adds all the functionality to the application. The click action to launch Google Maps, creating a message on clicking either check-box or the animal name is triggered by this class. The click action to launch Google Maps is achieved by a special type of object known as INTENT.

Intent intent $=$ new Intent (Intent.ACTION_VIEW, Uri.parse ("google.navigation: q=28.606057,

77. 243891\&mode=w"));

intent.setPackage("com.google.android.apps.ma ps");

Intent chooser $=$ Intent. createChooser (intent, "Open Location In Maps");

holder.btn.getContext().startActivity(chooser );

- RecyclerViewAdapterFav.java : This java class forms the recycler view for the Route Order list. This is the class that adds all the functionality to the application. The click action to launch Google Maps, creating a message on clicking either check-box or the animal name is triggered by this class. The click action to launch Google Maps is achieved by a special type of object known as INTENT.

Intent intent $=$ new Intent (Intent.ACTION_VIEW, Uri.parse("google.navigation: q=28.606057,

77.243891\&mode=w"));

intent.setPackage("com.google. android.apps.ma ps");

Intent chooser $=$ Intent.createChooser (intent, "Open Location In Maps");

holder.btn.getContext(). startActivity (chooser );

\section{RESULTS}

Using the application is very simple. The user opens the app and is shown two lists in two tabs. One list, lists the animals in $\mathrm{A}-Z$ order and the other lists the animal in Route Order. The user can reference any of the two lists according to his/her preference.

\begin{tabular}{|c|c|c|c|}
\hline \multicolumn{2}{|l|}{ Zoo } & \multicolumn{2}{|l|}{ Zoo } \\
\hline ROUTE & LER & ROUT & DER \\
\hline AFRICAN ELEPHANT & $\square$ & OSTRICH & $\square$ \\
\hline ASIATIC LION & $\square$ & EMU & $\square$ \\
\hline ASIATIC LION (2) & $\square$ & CASSOWARIES & ఐ \\
\hline BARKING DEER & $\square$ & REPTILE HOUSE & $\square$ \\
\hline BLACK BUCK & $\square$ & GAUR (INDIAN BISON) & ص \\
\hline BONNET MACAQUE & $\square$ & ROYAL BENGAL TIGER & ص \\
\hline $\begin{array}{l}\text { BROW-ANTLERED DEER } \\
\text { (SANGAI DEER) }\end{array}$ & $\square$ & WATER BIRDS & $\square$ \\
\hline CAPE BUFFALO & $\square$ & HIMALAYAN BLACK BEA & ص \\
\hline
\end{tabular}

Figure 4: Home of the Application

Let's say, the user wishes to visit "White Tiger" next. For that, he clicks on the White Tiger option. After clicking that option, a message is displayed saying 'Finding a path to White Tiger', then Google Maps is launched. Then, Google Maps finds the best route to the location of the White Tiger from the user's present location. 

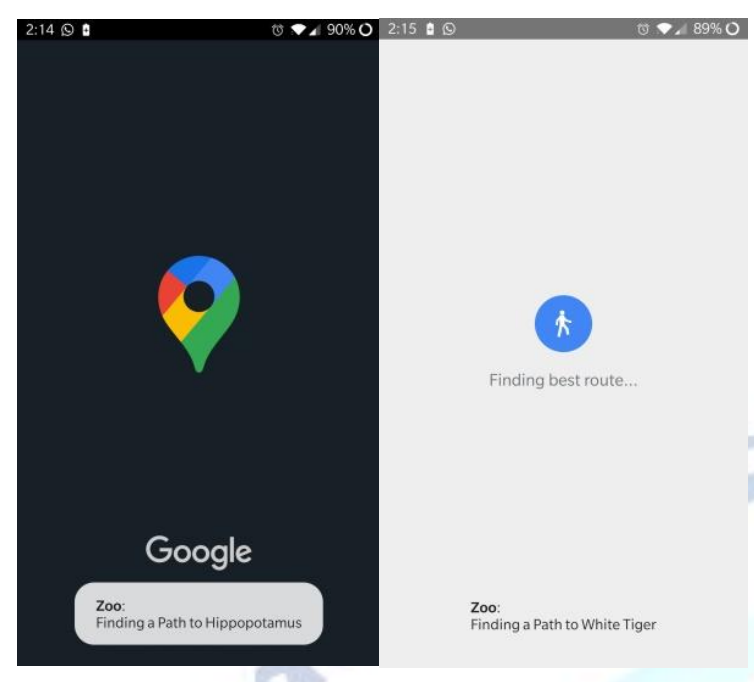

Figure 5: After clicking an animal's name

Then finally, the path to the location of the White Tiger is displayed by walking mode. The default mode is set to walking because the zoo visitors will travel inside the zoo by foot only.

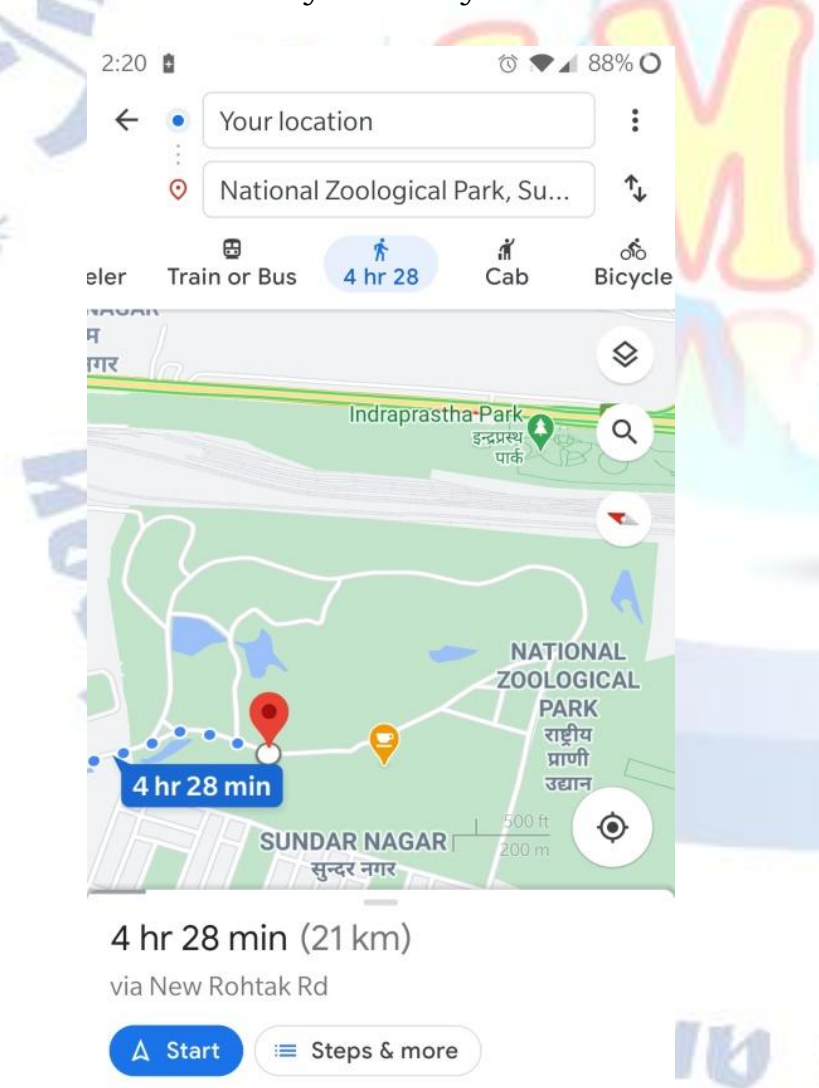

Figure 6: Path to animal by walking mode

The user can also click on the check-box next to an animal's name to mark it as visited. After clicking the check-box, a message will be displayed saying that that animal has been marked as visited.

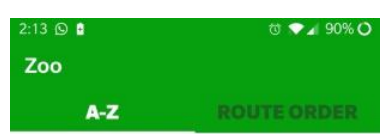

AFRICAN ELEPHANT

ASIATIC LION

ASIATIC LION (2)

BARKING DEER

BLACK BUCK

BONNET MACAQUE

BROW-ANTLERED DEER (SANGAI DEER)

C. Brow-Antlered Deer(Sangai Deer)

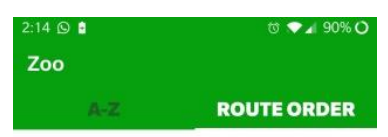

HIMALAYAN BLACK BEAR $\square$

HIMALAYAN BLACK BEAR (2)

SLOTH BEAR

GORAL

LEOPARD/PANTHER

CROCODILE

SPECTACLED CAIMAN

w $\underset{\text { Visited }}{\text { Himan Black Bear (2) Marked as }} \supset$
Figure 7: Message displayed on clicking the check-box

\section{CONCLUSION}

In this report, we have discussed about Android RecyclerView, its introduction, its advantages as well as disadvantages. We saw that in modern application development, RecyclerView provides much better functionality than ListView or GridView.

We also saw the basic implementation of RecyclerView. Also, the android application we discussed about works using RecyclerView and its various components.

In this application, the user is directed from the application to Google Maps application for which an INTENT object was used and the location of the animal was passed as the argument.

So, we can conclude that there is a great need for this application for not only the National Zoological Park but also all other amusement parks, theme parks, water parks, etc. A personalized application like this one can greatly increase the number of visitors per day because it will save significant amount of effort on the part of the user.

\section{REFERENCES}

[1] https://awsrh.blogspot.com/

[2] https://technical-coding.blogspot.com/

[3] https://www.google.com/maps

[4] https://www.zoochat.com/community/media/delhi -zoo-map-board.352344/

[5] https://en.wikipedia.org/wiki/National_Zoological_ Park_Delhi

[6] https://developer.android.com/docs

[7] https://levelup.gitconnected.com/android-recyclerview-b1bc493cac53 